56, 3, pp. 815-828, Warsaw 2018

DOI: $10.15632 /$ jtam-pl.56.3.815

\title{
PRIMARY PARAMETRIC RESONANCE OF AN AXIALLY ACCELERATING BEAM SUBJECTED TO STATIC LOADS
}

\author{
Yuda Hu, Yantian Rong \\ School of Civil Engineering and Mechanics, Yanshan University, Qinhuangdao, China \\ Hebei Provincial Key Laboratory of Mechanical Reliability for Heavy Equipments and Large Structures, Yanshan University, \\ Qinhuangdao, China \\ e-mail: huyuda03@163.com; Tiffany_rong@163.com
}

JING LI

School of Civil Engineering and Mechanics, Yanshan University, Qinhuangdao, China

Department of Basic Teaching, Tangshan University, Tangshan, China

e-mail: jingamanda721@163.com

\begin{abstract}
Primary parametric resonance and stability of an axially accelerating and current-carrying beam subjected to static loads in magnetic field are investigated. The nonlinear magneto-elastic vibration equation is derived. The approximate solution of the static problem and the disturbance deferential equation of the beam with two sides simply supported are obtained. The frequency-response equation of primary parametric resonance is further achieved by a multi-scale method. According to stability conditions, the stability of the steady-state solution is also discussed. By numerical examples, the amplitude versus different parameter curves and the bifurcation diagrams of the amplitude are acquired. The effects of magnetic induction intensity, axial speed, detuning parameter and static loads on nonlinear vibration characteristics are also analyzed.
\end{abstract}

Keywords: current-carrying beam, primary parametric resonance, magnetic field, axial movement, static loads

\section{Introduction}

In modern engineering life, with the rapid development of science and technology and wide application of axially moving systems, electromagnetic structures have been gradually applied in many significant industrial fields, for instance, aerospace and heavy-duty machinery. When interfered by electromagnetic field, mechanical field and parametric resonance, magneto-elastic structures may cause large amplitude vibration or even result in the loss of stability. Therefore, it is important to analyze nonlinear dynamic characteristics of axially accelerating beams in magnetic field when the beams are under coupled vibration. The nonlinear vibration model of electroconductive plate in the magnetic field has been established (Hasanyan et al., 2001) and the investigation of vibrational behaviors have also been made by means of the Hamilton principle and multi-scale method (Hasanyan et al., 2005). The dynamic stability and nonlinear subharmonic resonance of conductive plates under magnetic field have been investigated (Zheng et al., 2005; Hu and Li, 2009). Wang et al. (2003) analyzed magneto-thermo-elastic instability of simply supported ferromagnetic plates subjected to thermal and magnetic loadings and investigated effects of thermal and magnetic fields in some detail. Hu et al. (2015) analyzed the strong nonlinear subharmonic resonance of an axially moving plate and employed the singularity theory to analyze the corresponding transition variety and the effects of parameters on system bifurcation. Ghayesh and Balar (2008) studied the stability condition of the Rayleigh beam by 
the Routh-Hurwitz criterion. Non-linear parametric vibration and stability of an axially moving Timoshenko beam with two dynamic models were investigated by Ghayesh and Balar (2010). Principal parametric resonance of an axially accelerating viscoelastic beam was studied with two models: one was partial differential nonlinear model and the other an integro-partial differential nonlinear model (Chen and Yang, 2005). Chakraborty and Mallik (1998) investigated the effects of the parametrically excited nonlinear moving beam with and without an external harmonic excitation. Based on the Timoshenko model, parametric resonance of axially moving beams and dynamic stability of a viscoelastic variable motion beam were studied (Tang et al., 2009; Chen et al., 2010). Hu and Zhang (2013) analyzed the primary parametric resonance of a rectangular thin plate in magnetic field and the effect of different parameters on dynamic behaviors. The nonlinear resonance of a rotating circular plate with initial deflection in magnetic field was studied by Hu and Wang (2015). Wang and Chen (1998) applied the Galerkin integral method to obtain the differential equation of flexible circular plates and discussed the influence of initial deflection on vibration characteristics. The effect of initial deflections on natural vibration frequencies of shells was analyzed by Matsner (1978). However, these investigations are all limited to primary parametric resonance of current-carrying structures with initial deflection. Thus it is still imperative to understand nonlinear dynamic behaviors of axially accelerating structures subjected to static loads under magnetic field.

In this article, the primary parametric resonance of an axially accelerating and current-carrying beam subject to static loads under magnetic field is investigated and the stability of the steady-state solution is also discussed. Detailed numerical examples are employed to demonstrate that the system presents typical nonlinear vibration characteristics and complex dynamic behaviors.

\section{Magneto-elastic vibration equations of the axially moving and current-carrying beam}

An isotropic current-carrying beam under a magnetic field $\mathbf{B}=\left[0, B_{0 y}, 0\right]$, a uniformly distributed axial tension $F_{0 x}$ and a uniformly distributed transverse load $P_{z}$, travels along the centroidal axis $x$-direction with an axial speed $C$. As shown in Fig. 1 , the beam with length $l$, width $b$, thickness $h$ and mass density $\rho$ is charged with the electric current density vector $\mathbf{J}_{e}=\left[J_{0 x}(t), 0,0\right]$. And $t$ is time variable.

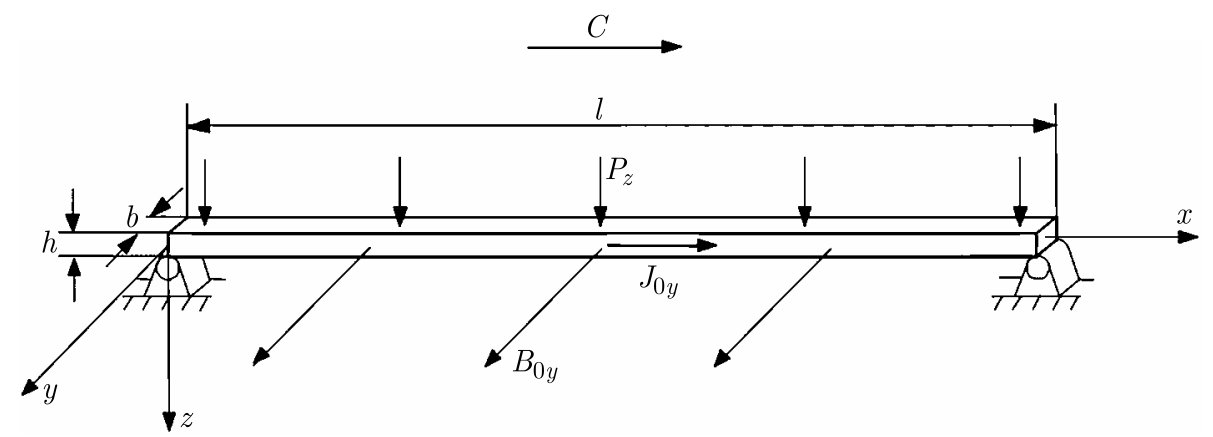

Fig. 1. Mechanical model of an axially accelerating and current-carrying beam in magnetic field

\subsection{Kinetic energy}

The transverse velocity of the axially accelerating beam can be expressed as follows

$$
V_{0 z}=\frac{d w}{d t}=\frac{\partial w}{\partial t}+C \frac{\partial w}{\partial x}
$$

where $w(x, t)$ is the transverse displacement. 
For this system, the total kinetic energy $T$ is

$$
T=\frac{1}{2} \rho \int_{-\frac{h}{2}}^{\frac{h}{2}} \int_{-\frac{b}{2}}^{\frac{b}{2}} \int_{0}^{l}\left(C^{2}+V_{0 z}^{2}\right) d x d y d z=\frac{1}{2} \rho A \int_{0}^{l}\left[C^{2}+\left(\frac{\partial w}{\partial t}+C \frac{\partial w}{\partial x}\right)^{2}\right] d x
$$

where $A=b \times h$ is the cross-sectional area of the beam.

\subsection{Potential energy}

According to the Euler-Bernoulli beam theory, the total potential energy of the beam is composed of three parts, namely, the strain potential energy $U_{1}$ induced by axial tension, the bending strain potential energy $U_{2}$ and the in-plane strain potential energy $U_{3}$. The total potential energy of the beam can be represented as follows

$$
\begin{aligned}
U & =U_{1}+U_{2}+U_{3} \\
& =\int_{0}^{l} F_{0 x} \varepsilon_{x} d x+\frac{1}{2} \int_{-\frac{h}{2}}^{\frac{h}{2}} \int_{-\frac{b}{2}}^{\frac{b}{2}} \int_{0}^{l} E\left(-\frac{\partial^{2} w}{\partial x^{2}} z\right)^{2} d x d y d z+\frac{1}{2} \int_{-\frac{h}{2}}^{\frac{h}{2}} \int_{-\frac{b}{2}}^{\frac{b}{2}} \int_{0}^{l} E \varepsilon_{x}^{2} d x d y d z \\
& =\int_{0}^{l}\left[F_{0 x} \varepsilon_{x}+\frac{1}{2} E I\left(\frac{\partial^{2} w}{\partial x^{2}}\right)^{2}+\frac{1}{2} E A \varepsilon_{x}^{2}\right] d x
\end{aligned}
$$

where $\varepsilon_{x}=(\partial w / \partial x)^{2} / 2$ is the normal strain component of the beam, $E$ is Young's modulus, $I=\int_{-h / 2}^{h / 2} \int_{-b / 2}^{b / 2} z^{2} d y d z$ is the cross sectional moment of inertia.

\subsection{Virtual work by the external force}

Transverse external forces acting on the beam include the transverse uniformly distributed forced excitation $P_{z}$ and electromagnetic force $F_{z}$. Hence, the virtual work generated by $P_{z}$ can be expressed as

$$
\delta W_{1}=\int_{0}^{l} P_{z} \delta w d x
$$

Neglecting the effect of magnetization and displacement current, the Lorentz force of a good conductor can be expressed as

$$
\mathbf{f}=\mathbf{J} \times \mathbf{B}
$$

where $\mathbf{J}$ is the electric current density in the beam.

Equation (2.5) can be rewritten as below

$$
\mathbf{f}=\mathbf{J}_{0 x} \times \mathbf{B}+\mathbf{J}_{x} \times \mathbf{B}=\left|\begin{array}{ccc}
\mathbf{i} & \mathbf{j} & \mathbf{k} \\
J_{0 x}+J_{x} & 0 & 0 \\
0 & B_{0 y} & 0
\end{array}\right|=\left(J_{0 x} B_{0 y}+J_{x} B_{0 y}\right) \mathbf{k}
$$

where $\mathbf{i}, \mathbf{j}$, and $\mathbf{k}$ are the unit vectors in the $x, y$, and $z$ directions, respectively, $J_{x}=-\sigma_{0} V_{0 z} B_{0}$ is the induced current density component in the conductive beam due to the external magnetic field along the $x$ axis, and $\sigma_{0}$ is the electric conductivity. 
The electromagnetic force per unit length can be derived from Eq. (2.6)

$$
F_{z}=\int_{-\frac{h}{2}}^{\frac{h}{2}} \int_{-\frac{b}{2}}^{\frac{b}{2}}\left(J_{0 x} B_{0 y}+J_{x} B_{0 y}\right) d y d z
$$

So. the virtual work due to the electromagnetic force can be represented as

$$
\delta W_{2}=\int_{0}^{l} F_{z} \delta w d x
$$

\subsection{Establishing the vibration equation by the Hamilton principle}

Based on the Hamilton principle, which is one of the most important integral principles of mechanics, one can get

$$
\int_{t_{1}}^{t_{2}}\left(\delta T-\delta U+\delta W_{1}+\delta W_{2}\right) d t=0
$$

where $\delta T$ is the variational expression of kinetic energy and $\delta U$ is the variational expression of potential energy.

Substitution of Eqs. (2.2), (2.3), (2.4) and (2.8) into Eq. (2.9) yields the nonlinear magneto-elastic vibration equation of the axially accelerating beam

$$
\begin{gathered}
\rho A \frac{\partial^{2} w}{\partial t^{2}}+2 \rho A C \frac{\partial^{2} w}{\partial x \partial t}+\rho A C^{2} \frac{\partial^{2} w}{\partial x^{2}}+\rho A \frac{\partial C}{\partial t} \frac{\partial w}{\partial x}-F_{0 x} \frac{\partial^{2} w}{\partial x^{2}} \\
-\frac{3}{2} E A\left(\frac{\partial w}{\partial x}\right)^{2} \frac{\partial^{2} w}{\partial x^{2}}+E I \frac{\partial^{4} w}{\partial x^{4}}=F_{z}+P_{z}
\end{gathered}
$$

where

$$
F_{z}=A B_{0 y} J_{0 x}-A \sigma_{0} B_{0 y}^{2}\left(\frac{\partial w}{\partial t}+C \frac{\partial w}{\partial x}\right)
$$

\section{Disturbance differential equation of the axially accelerating beam subjected to static loads}

When a system is under a constant forced excitation $\left(P_{z}=P_{c}\right)$ and a constant magnetic induction intensity $\left(B_{0 y}=B_{0}\right)$ and charged with a direct current $\left(J_{0 x}=J_{c}\right)$, the value of $A B_{0 y} J_{0 x}$ is constant. Let $Q_{z}=A B_{0} J_{c}, Q=P_{c}+Q_{z}$, and $Q$ is a uniformly distributed static load. Equation (2.10) can be expressed in the following form

$$
\begin{aligned}
& \rho A \frac{\partial^{2} w}{\partial t^{2}}+2 \rho A C \frac{\partial^{2} w}{\partial x \partial t}+\rho A C^{2} \frac{\partial^{2} w}{\partial x^{2}}+\rho A \frac{\partial C}{\partial t} \frac{\partial w}{\partial x}-F_{0 x} \frac{\partial^{2} w}{\partial x^{2}}-\frac{3}{2} E A\left(\frac{\partial w}{\partial x}\right)^{2} \frac{\partial^{2} w}{\partial x^{2}} \\
& +E I \frac{\partial^{4} w}{\partial x^{4}}+A \sigma_{0} B_{0}^{2}\left(\frac{\partial w}{\partial t}+C \frac{\partial w}{\partial x}\right)=Q
\end{aligned}
$$

It is assumed that the beam has a tiny static deflection $w_{0}$ under the uniformly distributed static load $Q$ and a deflection $w_{1}$ when it vibrates. Therefore, the total deflection of the system can be given as below

$$
w=w_{0}+w_{1}
$$


Therefore, Eq. (3.1) can be rewritten as

$$
\begin{aligned}
\rho A & \frac{\partial^{2} w_{1}}{\partial t^{2}}+2 \rho A C \frac{\partial^{2} w_{1}}{\partial x \partial t}+\rho A C^{2}\left(\frac{\partial^{2} w_{0}}{\partial x^{2}}+\frac{\partial^{2} w_{1}}{\partial x^{2}}\right)+\rho A \frac{\partial C}{\partial t} \frac{\partial w_{1}}{\partial x} \\
& -F_{0 x}\left(\frac{\partial^{2} w_{0}}{\partial x^{2}}+\frac{\partial^{2} w_{1}}{\partial x^{2}}\right)-\frac{3}{2} E A\left(\frac{\partial w_{0}}{\partial x}+\frac{\partial w_{1}}{\partial x}\right)^{2}\left(\frac{\partial^{2} w_{0}}{\partial x^{2}}+\frac{\partial^{2} w_{1}}{\partial x^{2}}\right) \\
& +E I\left(\frac{\partial^{4} w_{0}}{\partial x^{4}}+\frac{\partial^{4} w_{1}}{\partial x^{4}}\right)+A \sigma_{0} B_{0}^{2}\left[\frac{\partial w_{1}}{\partial t}+C\left(\frac{\partial w_{0}}{\partial x}+\frac{\partial w_{1}}{\partial x}\right)\right]=Q
\end{aligned}
$$

The initial deflection $w_{0}$ satisfies the following equation

$$
\rho A C^{2} \frac{\partial^{2} w_{0}}{\partial x^{2}}-F_{0 x} \frac{\partial^{2} w_{0}}{\partial x^{2}}-\frac{3}{2} E A\left(\frac{\partial w_{0}}{\partial x}\right)^{2} \frac{\partial^{2} w_{0}}{\partial x^{2}}+E I \frac{\partial^{4} w_{0}}{\partial x^{4}}+A \sigma_{0} B_{0}^{2} C \frac{\partial w_{0}}{\partial x}=Q
$$

According to the axially moving beam simply supported on two sides, its boundary conditions are

$$
\begin{aligned}
x=0: & w=0 & \frac{\partial^{2} w}{\partial x^{2}}=0 \\
x=l: & w=0 & \frac{\partial^{2} w}{\partial x^{2}}=0
\end{aligned}
$$

Assume that the static deflection displacement satisfies the boundary condition

$$
w_{0}=f_{0} \sin \frac{\pi x}{l}
$$

After substituting Eq. (3.5) into Eq. (3.4) and using the Galerkin method, we can get

$$
\begin{gathered}
\int_{0}^{l}\left[\rho A C^{2} \frac{\partial^{2} w_{0}}{\partial x^{2}}-F_{0 x} \frac{\partial^{2} w_{0}}{\partial x^{2}}-\frac{3}{2} E A\left(\frac{\partial w_{0}}{\partial x}\right)^{2} \frac{\partial^{2} w_{0}}{\partial x^{2}}\right] \sin \frac{\pi x}{l} d x \\
+\int_{0}^{l}\left(E I \frac{\partial^{4} w_{0}}{\partial x^{4}}+A \sigma_{0} B_{0}^{2} C \frac{\partial w_{0}}{\partial x}-Q\right) \sin \frac{\pi x}{l} d x=0
\end{gathered}
$$

After integration, the following formula is obtained

$$
a_{2} f_{0}^{3}-\left(a_{3}-a_{1}\right) f_{0}+a_{4} Q=0
$$

where

$$
a_{1}=\frac{E I \pi^{4}}{2 l^{3}} \quad a_{2}=\frac{3 E A \pi^{4}}{16 l^{3}} \quad a_{3}=\frac{\rho A C^{2} \pi^{2}-F_{0 x} \pi^{2}}{2 l} \quad a_{4}=-\frac{2 l}{\pi}
$$

The real root of Eq. (3.7) is

$$
f_{0}=Z_{0}-\frac{a_{1}-a_{3}}{3 a_{2} Z_{0}}
$$

where

$$
Z_{0}=\sqrt[3]{\sqrt{\left(\frac{a_{1}-a_{3}}{3 a_{2}}\right)^{3}+\left(\frac{a_{4} Q}{2 a_{2}}\right)^{2}}-\frac{a_{4} Q}{2 a_{2}}}
$$


Considering Eq. (3.4) for the initial static deflection $w_{0}$ in Eq. (3.3), we can write the following equation for the deflection $w_{1}$

$$
\begin{aligned}
\rho A & \frac{\partial^{2} w_{1}}{\partial t^{2}}+2 \rho A C \frac{\partial^{2} w_{1}}{\partial x \partial t}+\rho A C^{2} \frac{\partial^{2} w_{1}}{\partial x^{2}}+\rho A \frac{\partial C}{\partial t} \frac{\partial w_{1}}{\partial x}-F_{0 x} \frac{\partial^{2} w_{1}}{\partial x^{2}} \\
& -\frac{3}{2} E A\left[2 \frac{\partial w_{0}}{\partial x} \frac{\partial w_{1}}{\partial x}\left(\frac{\partial^{2} w_{0}}{\partial x^{2}}+\frac{\partial^{2} w_{1}}{\partial x^{2}}\right)+\left(\frac{\partial w_{1}}{\partial x}\right)^{2} \frac{\partial^{2} w_{0}}{\partial x^{2}}+\left(\frac{\partial w_{0}}{\partial x}\right)^{2} \frac{\partial^{2} w_{1}}{\partial x^{2}}\right. \\
& \left.+\left(\frac{\partial w_{1}}{\partial x}\right)^{2} \frac{\partial^{2} w_{1}}{\partial x^{2}}\right]+E I \frac{\partial^{4} w_{1}}{\partial x^{4}}+A \sigma_{0} B_{0}^{2}\left(\frac{\partial w_{1}}{\partial t}+C \frac{\partial w_{1}}{\partial x}\right)=0
\end{aligned}
$$

For the axially accelerating beam, its axial speed and axial tension can be assumed as follows, respectively

$$
C=C_{0}+C_{1} \cos \left(\omega_{1} t\right) \quad F_{0 x}=F_{0}+F_{1} \cos \left(\omega_{2} t\right)
$$

According to the simply supported boundary condition, the deflection $w_{1}$ can be denoted as

$$
w_{1}=s(t) \sin \frac{\pi x}{l}
$$

where $C_{0}$ is the axial constant speed, $C_{1}$ is the amplitude of the time-variant axial speed, $F_{0}$ is the axial constant tension, $F_{1}$ is the amplitude of the time-variant axial tension, and $\omega_{1}$ and $\omega_{2}$ are the frequencies of the time-variant axial speed and time-variant axial tension, respectively.

Substituting Eqs. (3.10) and (3.11) into Eq. (3.9) and using the Galerkin method yields the dimensionless magneto-elastic parametric vibration differential equation

$$
\ddot{q}(\tau)+2 \widetilde{\zeta} \dot{q}(\tau)+\omega_{0}^{2} q(\tau)-\left[\widetilde{k}_{1} \cos (2 \tau)+\widetilde{k}_{2} \cos (4 \tau)-\widetilde{k}_{3} \cos (2 \tau)\right] q(\tau)-\widetilde{\alpha}_{4} q^{2}(\tau)+\widetilde{\alpha}_{3} q^{3}(\tau)=0
$$

where

$$
\begin{aligned}
& q=\frac{s}{h} \quad 2 \tau=\omega_{1} t \quad 2 \tau=\omega_{2} t \quad \widetilde{\zeta}=\frac{\sigma_{0} B_{0}^{2}}{\rho \omega_{1}} \\
& \widetilde{k}_{1}=\frac{8 C_{0} C_{1} \pi^{2}}{\omega_{1}^{2} l^{2}} \quad \widetilde{k}_{2}=\frac{2 C_{1}^{2} \pi^{2}}{\omega_{1}^{2} l^{2}} \quad \widetilde{k}_{3}=\frac{4 \pi^{2} F_{1}}{\rho A \omega_{1}^{2} l^{2}} \\
& \omega_{0}^{2}=\frac{4 \pi^{2} F_{0} l^{2}+4 \pi^{4} E I+\frac{9}{2} E A \pi^{4} f_{0}^{2}-2 \rho A l^{2} \pi^{2} C_{1}^{2}-4 \rho A l^{2} \pi^{2} C_{0}^{2}}{\rho A l^{4} \omega_{1}^{2}} \\
& \widetilde{\alpha}_{4}=\frac{9 f_{0} E \pi^{4} h}{2 \rho \omega_{1}^{2} l^{4}} \quad \widetilde{\alpha}_{3}=\frac{3 E \pi^{4} h^{2}}{2 \rho \omega_{1}^{2} l^{4}}
\end{aligned}
$$

\section{Solving the primary parametric vibration problem by the method of multiple scales}

In order to analyze the problem of principal parametric resonance of Eq. (3.12), a small parameter $\varepsilon$ is introduced

$$
\ddot{q}(\tau)+2 \varepsilon \zeta \dot{q}(\tau)+\omega_{0}^{2} q(\tau)-\varepsilon\left[k_{1} \cos (2 \tau)+k_{2} \cos (4 \tau)-k_{3} \cos (2 \tau)\right] q(\tau)-\varepsilon \alpha_{4} q^{2}(\tau)+\varepsilon \alpha_{3} q^{3}(\tau)=0
$$

where

$$
\zeta=\frac{\widetilde{\zeta}}{\varepsilon} \quad k_{1}=\frac{\widetilde{k}_{1}}{\varepsilon} \quad k_{2}=\frac{\widetilde{k}_{2}}{\varepsilon} \quad k_{3}=\frac{\widetilde{k}_{3}}{\varepsilon} \quad \alpha_{3}=\frac{\widetilde{\alpha}_{3}}{\varepsilon} \quad \alpha_{4}=\frac{\widetilde{\alpha}_{4}}{\varepsilon}
$$


When solving the problem of the principal parametric resonance, we let

$$
\omega_{0}=1+\varepsilon \sigma
$$

where $\sigma$ is the detuning parameter.

The first-order approximate solution to the vibration differential equation can be found through the method of multiple scales (Nayfeh and Mook, 1979). Meanwhile, the fast time scale $T_{0}=\tau$ and the low time $T_{1}=\varepsilon \tau$ scale are introduced. The approximate analytical solution of parametric resonance can be drawn as

$$
q(\tau, \varepsilon)=q_{0}\left(T_{0}, T_{1}\right)+\varepsilon q_{1}\left(T_{0}, T_{1}\right)
$$

Substituting Eqs. (4.2) and (4.3) into Eq. (4.1), and equating the coefficients of $\varepsilon^{0}$ and $\varepsilon^{1}$ on both sides, one can conclude that

$$
\begin{aligned}
& D_{0}^{2} q_{0}+q_{0}=0 \\
& D_{0}^{2} q_{1}^{2}+q_{1}=-2 D_{0} D_{1} q_{0}-2 \zeta D_{0} q_{0}-2 \sigma q_{0} \\
& \quad+\left[k_{1} \cos (2 \tau)+k_{2} \cos (4 \tau)-k_{3} \cos (2 \tau)\right] q_{0}-\alpha_{3} q_{0}^{3}+\alpha_{4} q_{0}^{2}
\end{aligned}
$$

where $D_{0}=\partial / \partial T_{0}$ and $D_{1}=\partial / \partial T_{1}$.

The general solution of zero-order approximate Eq. $(4.4)_{1}$ can be expressed as follow

$$
q_{0}=A_{0}\left(T_{1}\right) \mathrm{e}^{\mathrm{i} T_{0}}+\bar{A}_{0}\left(T_{1}\right) \mathrm{e}^{-\mathrm{i} T_{0}}
$$

where $\mathrm{i}^{2}=-1, A_{0}$ is an unknown complex number and $\bar{A}_{0}$ is the conjugate complex of $A_{0}$. Substitution of Eq. (4.5) into first-order approximate Eq. $(4.4)_{2}$ gives

$$
\begin{gathered}
D_{0}^{2} q_{1}^{2}+q_{1}=-2 \mathrm{i} A_{0}^{\prime} \mathrm{e}^{\mathrm{i} T_{0}}-2 \mathrm{i} \zeta A_{0} \mathrm{e}^{\mathrm{i} T_{0}}-2 \sigma A_{0} \mathrm{e}^{\mathrm{i} T_{0}}-3 \alpha_{3} A_{0}^{2} \bar{A} \mathrm{e}^{\mathrm{i} T_{0}}+\alpha_{4} A_{0}^{2} \mathrm{e}^{\mathrm{i} 2 T_{0}}+A_{0} \bar{A}_{0} \\
-\alpha_{3} A_{0}^{3} \mathrm{e}^{\mathrm{i} 3 T_{0}}+\frac{k_{1}-k_{3}}{2}\left(A_{0} \mathrm{e}^{\mathrm{i} 3 T_{0}}+\bar{A}_{0} \mathrm{e}^{\mathrm{i} T_{0}}\right)+\frac{k_{2}}{2}\left(A_{0} \mathrm{e}^{\mathrm{i} 5 T_{0}}+\bar{A}_{0} \mathrm{e}^{\mathrm{i} 3 T_{0}}\right)+c c
\end{gathered}
$$

where $A_{0}^{\prime}=\partial A_{0} / \partial T_{1}, c c$ stands for the conjugate complex part of the function on the right-hand side of Eq. (4.6).

Eliminating the secular term from the particular solution to Eq. (4.6)

$$
-2 \mathrm{i} A_{0}^{\prime}-2 \mathrm{i} \zeta A_{0}-2 \sigma A_{0}-3 \alpha_{3} A_{0}^{2} \bar{A}_{0}+\frac{k_{1}-k_{3}}{2} \bar{A}_{0}=0
$$

Express now $A_{0}$ of Eq. (4.7) in the polar form

$$
A_{0}=\frac{1}{2} a\left(T_{1}\right) \mathrm{e}^{\mathrm{i} \varphi\left(T_{1}\right)}
$$

where $a$ and $\varphi$ are real.

Substituting Eq. (4.8) into Eq. (4.7) we separate the conclusion into its real and imaginary parts, and arrive at

$$
a^{\prime}=-\zeta a+\frac{k_{1}-k_{3}}{4} a \sin (2 \varphi) \quad a \varphi^{\prime}=\sigma a+\frac{3 \alpha_{3}}{8} a^{3}-\frac{k_{1}-k_{3}}{4} a \cos (2 \varphi)
$$

There are $a^{\prime}=0$ and $\varphi^{\prime}=0$ according to steady state motion of the system. So, we conclude

$$
\zeta a=\frac{k_{1}-k_{3}}{4} a \sin (2 \varphi) \quad \sigma a+\frac{3 \alpha_{3}}{8} a^{3}=\frac{k_{1}-k_{3}}{4} a \cos (2 \varphi)
$$

The frequency-response equation is achieved according to Eqs. (4.10)

$$
(\zeta a)^{2}+\left(\sigma a+\frac{3 \alpha_{3}}{8} a^{3}\right)^{2}=\left(\frac{k_{1}-k_{3}}{4} a\right)^{2}
$$




\section{Stability analysis of steady state motion}

We determine the stability of steady-state motion by investigating the nature of the singular points of Eqs. (4.9). To accomplish this, we let

$$
a=a_{0}+a_{s} \quad \varphi=\varphi_{0}+\varphi_{s}
$$

where $a_{0}$ and $\varphi_{0}$ are equilibrium solutions of the steady motion, $a_{s}$ and $\varphi_{s}$ are tiny disturbance values.

Substituting Eqs. (5.1) into Eqs. (4.9), expanding for small $a_{s}$ and $\varphi_{s}$, noting that $a_{s}$ and $\varphi_{s}$ satisfy Eqs. (4.10) and keeping linear in $a_{s}$ and $\varphi_{s}$, we obtain

$$
a_{s}^{\prime}=-2 a_{0}\left(\sigma+\frac{3 \alpha_{3}}{8} a^{2}\right) \varphi_{s} \quad \varphi_{s}^{\prime}=\frac{3 \eta_{3} a_{0}}{4} a_{s}-2 \zeta \varphi_{s}
$$

Thus, the stability of steady-state motions depends on the eigenvalues of the coefficient matrix on the right-hand sides of Eqs. (5.2). Using Eqs. (4.10), one can obtain the following eigenvalue equation

$$
\left|\begin{array}{cc}
-\lambda & -2 a_{0}\left(\sigma+\frac{3 \eta_{3}}{8} a_{0}^{2}\right) \\
\frac{3 \eta_{3} a_{0}}{4} & -2 \zeta-\lambda
\end{array}\right|=0
$$

The solution of the system is stable only if the real parts of the eigenvalues are negative according to the stability theory. Based on the Routh-Hurwitz criteria, we conclude

$$
\frac{3 \alpha_{3}}{2} a_{0}^{2}\left(\sigma+\frac{3 \alpha_{3}}{8} a_{0}^{2}\right)>0
$$

\section{Analysis of numerical illustrations}

The numerical results are based on a copper beam model with length $l=0.3 \mathrm{~m}$, width $b=0.02 \mathrm{~m}$, thickness $h=0.01 \mathrm{~m}$, Young's modulus $E=108 \mathrm{GPa}$, mass density $\rho=8920 \mathrm{~kg} / \mathrm{m}^{3}$, Poisson's ratio $\mu=0.33$, and electric conductivity $\sigma_{0}=5.7143 \cdot 10^{7}(\Omega \cdot \mathrm{m})^{-1}$. Figures $2-7$ represent the response curve of amplitude $a$ versus the detuning parameter $\varepsilon \sigma$, the amplitude of time-variant axial tension $F_{1}$ and the static load $Q$. The solid lines represent the stable solutions and the dotted lines represent the unstable solutions. Figure 8 demonstrates the bifurcation diagram of the amplitude.

\subsection{The curve of the amplitude-detuning parameter}

For the given range in Fig. 2 in which the detuning parameter $\varepsilon \sigma$ changes from negative to positive, the amplitude $a$ drops gradually to zero. The unstable solutions have tend to decrease as the axial constant velocity $C_{0}$, the amplitude of the time-variant axial speed $C_{1}$, the amplitude of the time-variant axial tension $F_{1}$ increase and the magnetic induction intensity $B_{0}$, the static load $Q$ decrease. It is also noted that the resonance region between the unstable solutions and the stable solutions broadens as $C_{0}, C_{1}$ and $F_{1}$ increase and $B_{0}$ decreases. In Figs. 2a and 2e, the curves intersect and have different variation on both sides of the intersection point due to the value of $k_{1}-k_{3}$ and $\omega_{0}$ varying with $C_{0}$. An increase of the static load $Q$ leads an increase of $\omega_{0}$ (Eqs. (3.8) and (3.12)). In Figs. 2b, 2c and 2d, we can note that the amplitudes of the stable solutions increase as $C_{1}$ and $F_{1}$ go up and $B_{0}$ comes down. 
(a)

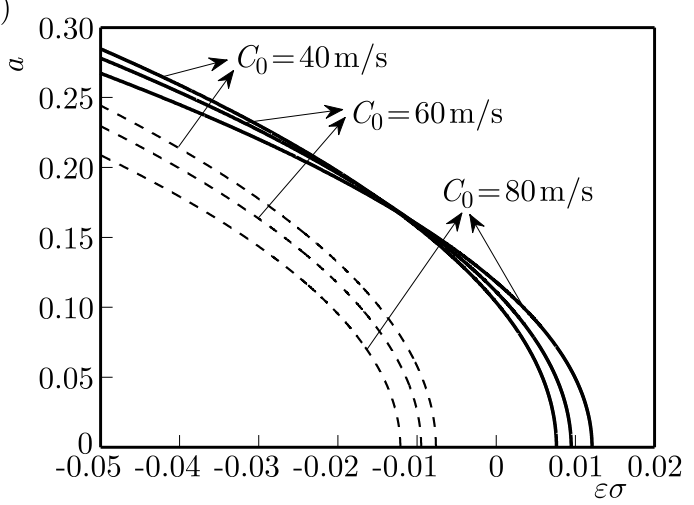

(c)

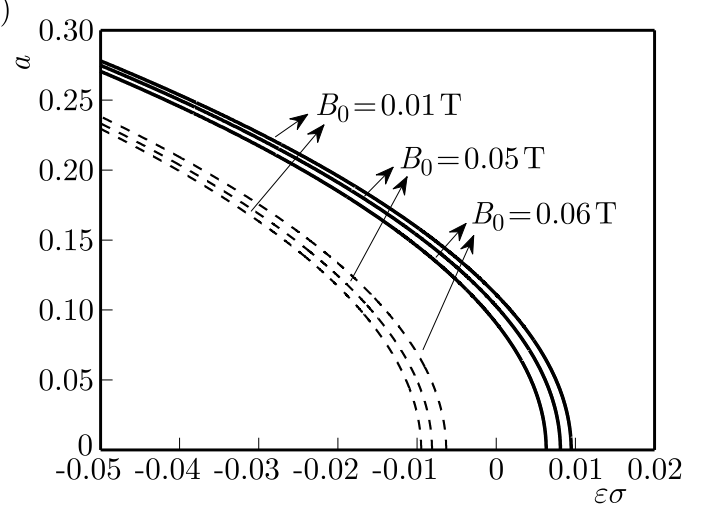

(b)

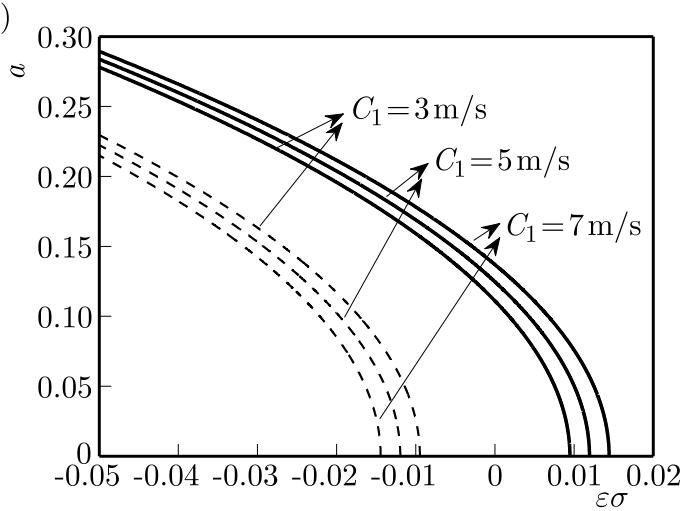

(d)

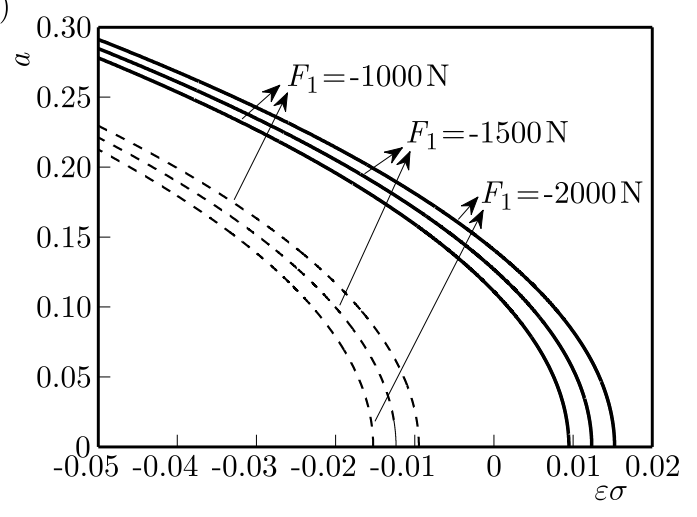

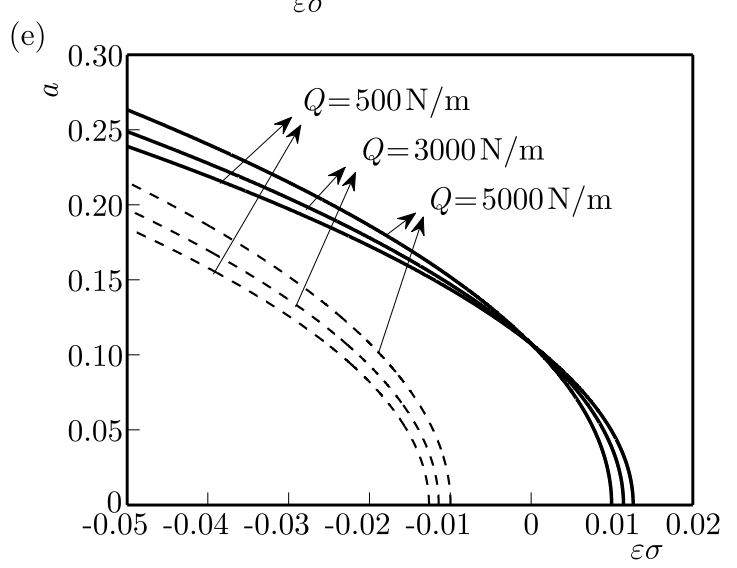

Fig. 2. The curve of amplitude frequency, $J_{c}=0.02 \mathrm{~A} / \mathrm{mm}^{2}$ :

(a) $C_{1}=3 \mathrm{~m} / \mathrm{s}, B_{0}=0.01 \mathrm{~T}, F_{1}=-1000 \mathrm{~N}$ when $P_{c}=0 \mathrm{~N} / \mathrm{m}$ and $F_{0}=30 \mathrm{kN}$;

(b) $C_{0}=60 \mathrm{~m} / \mathrm{s}, B_{0}=0.01 \mathrm{~T}, F_{1}=-1000 \mathrm{~N}$ when $P_{c}=0 \mathrm{~N} / \mathrm{m}$ and $F_{0}=30 \mathrm{kN}$;

(c) $C_{0}=60 \mathrm{~m} / \mathrm{s}, C_{1}=3 \mathrm{~m} / \mathrm{s}, F_{1}=-1000 \mathrm{~N}$ when $P_{c}=0 \mathrm{~N} / \mathrm{m}$ and $F_{0}=30 \mathrm{kN}$;

(d) $C_{0}=60 \mathrm{~m} / \mathrm{s}, C_{1}=3 \mathrm{~m} / \mathrm{s}, B_{0}=0.01 \mathrm{~T}$ when $P_{c}=0 \mathrm{~N} / \mathrm{m}$ and $F_{0}=30 \mathrm{kN}$;

(e) $C_{0}=50 \mathrm{~m} / \mathrm{s}, C_{1}=3 \mathrm{~m} / \mathrm{s}, B_{0}=0.01 \dot{\mathrm{T}}, F_{1}=-1000 \mathrm{~N}$ when $F_{0}=15 \mathrm{kN}$

\subsection{The curve of the amplitude-parametric excitation}

Figure 3 represents the response curve of the amplitude $a$ versus the parametric excitation $F_{1}$ under the influence of magnetic induction intensity $B_{0}$. The zero solution region becomes wide when the magnetic induction intensity $B_{0}$ goes up, that is, the non-resonance region broadens. From Fig. 3, we note that the system has stable nontrivial solutions when the detuning parameter $\varepsilon \sigma=0$, and the unstable nontrivial solutions when the detuning parameter $\varepsilon \sigma \neq 0$. All curves in Fig. 3 are symmetrically distributed with $F_{0}=0 \mathrm{~N} / \mathrm{m}$ line when the amplitude of the time-variant axial speed $C_{1}=0 \mathrm{~m} / \mathrm{s}$. When $C_{1}=1 \mathrm{~m} / \mathrm{s}$, the symmetry axis shifts towards right. 
(a)

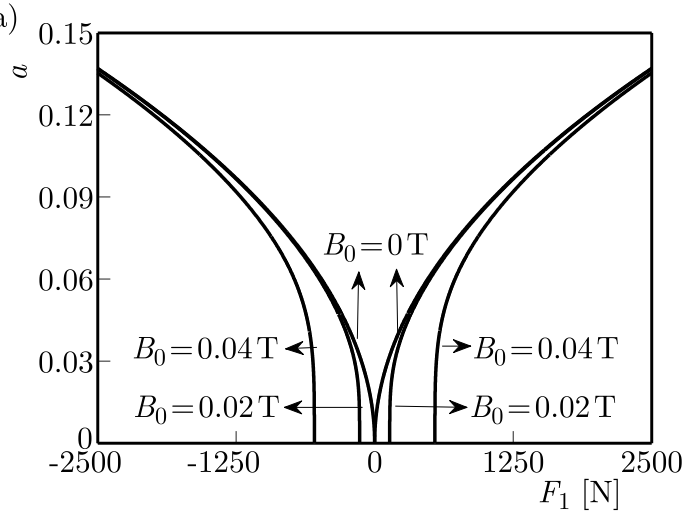

(c)

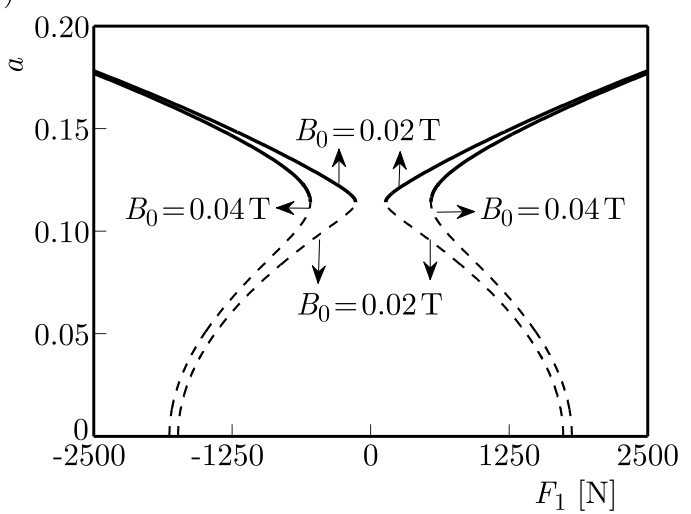

(b)

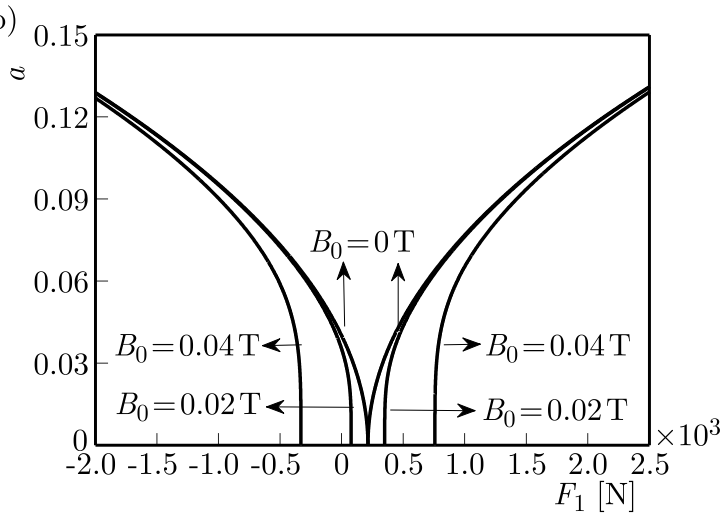

(d)

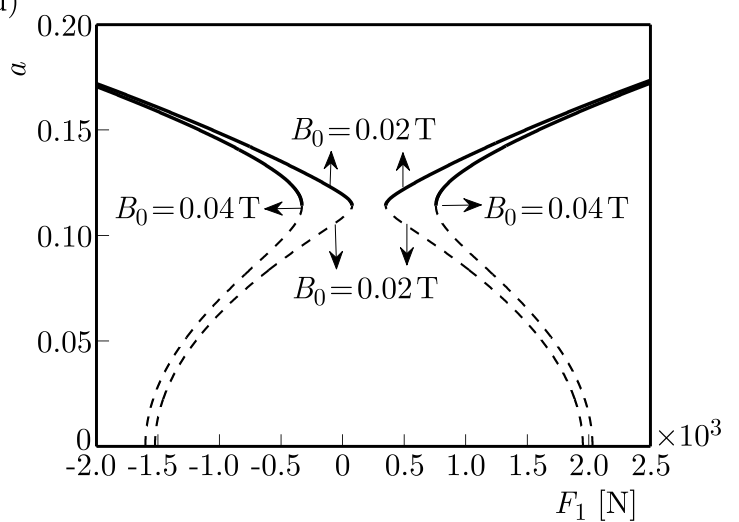

Fig. 3. The curve of the amplitude-parametric excitation, $F_{0}=30 \mathrm{kN}, J_{c}=0.02 \mathrm{~A} / \mathrm{mm}^{2}, P_{c}=0 \mathrm{~N} / \mathrm{m}$ : (a) $\varepsilon \sigma=0, C_{0}=60 \mathrm{~m} / \mathrm{s}, C_{1}=0 \mathrm{~m} / \mathrm{s} ;$ (b) $\varepsilon \sigma=0, C_{0}=60 \mathrm{~m} / \mathrm{s}, C_{1}=1 \mathrm{~m} / \mathrm{s}$;

(c) $\varepsilon \sigma=-0.01, C_{0}=60 \mathrm{~m} / \mathrm{s}, C_{1}=0 \mathrm{~m} / \mathrm{s} ;$ (d) $\varepsilon \sigma=-0.01, C_{0}=60 \mathrm{~m} / \mathrm{s}, C_{1}=1 \mathrm{~m} / \mathrm{s}$

According to the frequency-response equation, the square item in the right-side contains $F_{1}$. Due to $F_{1}$ varying from a positive to a negative value, the curves present symmetrical distribution. And the existence of the time-variant axial speed can cause the curves shift towards right. Similar phenomena are also observed in Figs. 4-6.

Figure 4 represents the response curve of the amplitude $a$ versus the parametric excitation $F_{1}$ under the influence of the amplitude of the time-variant axial speed $C_{1}$. In Fig. 4, for the stable solutions on the left side, the greater $C_{1}$ is, the larger the vibration amplitude $a$ becomes. However, it has the opposite result on the right side.

Figure 5 represents the response curve of the amplitude $a$ versus the parametric excitation $F_{1}$ under the influence of the detuning parameter $\varepsilon \sigma$. In Figs. 5a, $5 \mathrm{~b}$ and $5 \mathrm{c}$, as the absolute value of the detuning parameter $\varepsilon \sigma$ gets greater, the vibration amplitude $a$ becomes larger. In Figs. $5 \mathrm{~d}$, $5 \mathrm{e}$ and $5 \mathrm{f}$, with a gradual increase of the magnetic induction intensity $B_{0}$, the zero solution region appears and gets broader.

Figure 6 represents the response curve of the amplitude $a$ versus the parametric excitation $F_{1}$ under the influence of the static load $Q$. Figure 6a shows that the increasing static load $Q$ makes the vibration amplitude $a$ decrease. However, the tendency in Fig. 6b is opposite.

\subsection{The curve of amplitude - static load}

Figure 7 shows that the system has only stable nontrivial solutions, and the increasing static load $Q$ decreases the vibration amplitude $a$. We can also see that the vibration amplitude $a$ is increasing when the axial constant velocity $C_{0}$, the amplitude of time-variant axial speed $C_{1}$, the amplitude of the time-variant axial tension $F_{1}$ increase and the magnetic induction intensity $B_{0}$ 
(a)

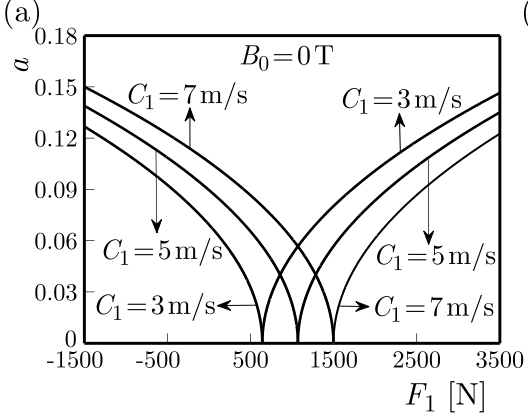

(d)

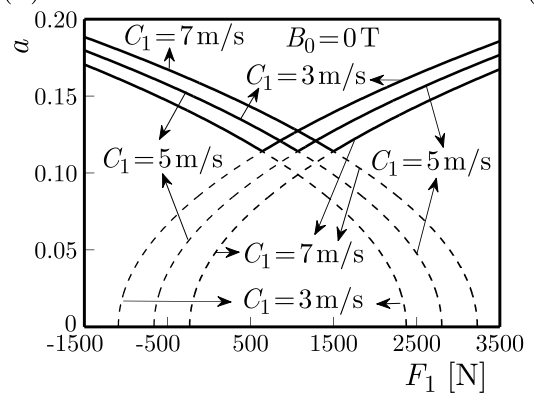

(b)

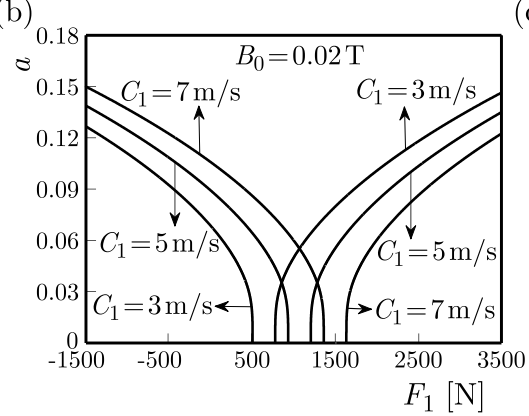

(e)

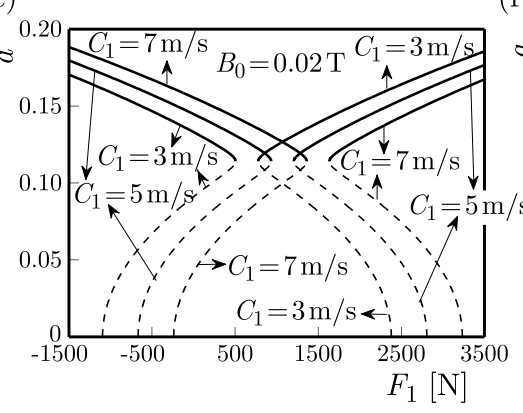

(c)

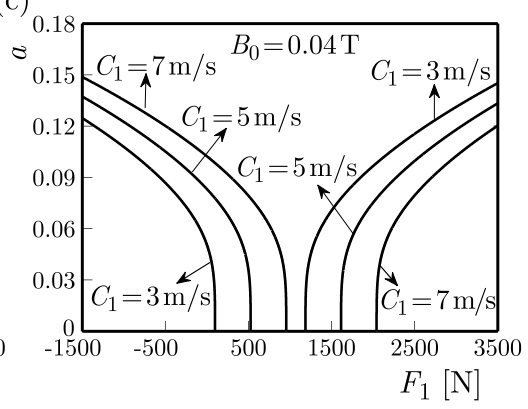

(f)

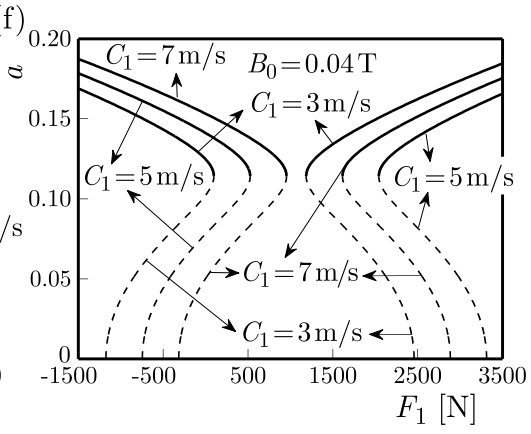

Fig. 4 . The curve of the amplitude-parametric excitation, $F_{0}=30 \mathrm{kN}, J_{c}=0.02 \mathrm{~A} / \mathrm{mm}^{2}, P_{c}=0 \mathrm{~N} / \mathrm{m}$ : (a) $\varepsilon \sigma=0, C_{0}=60 \mathrm{~m} / \mathrm{s}$; (b) $\varepsilon \sigma=-0.01, C_{0}=60 \mathrm{~m} / \mathrm{s}$

(a)
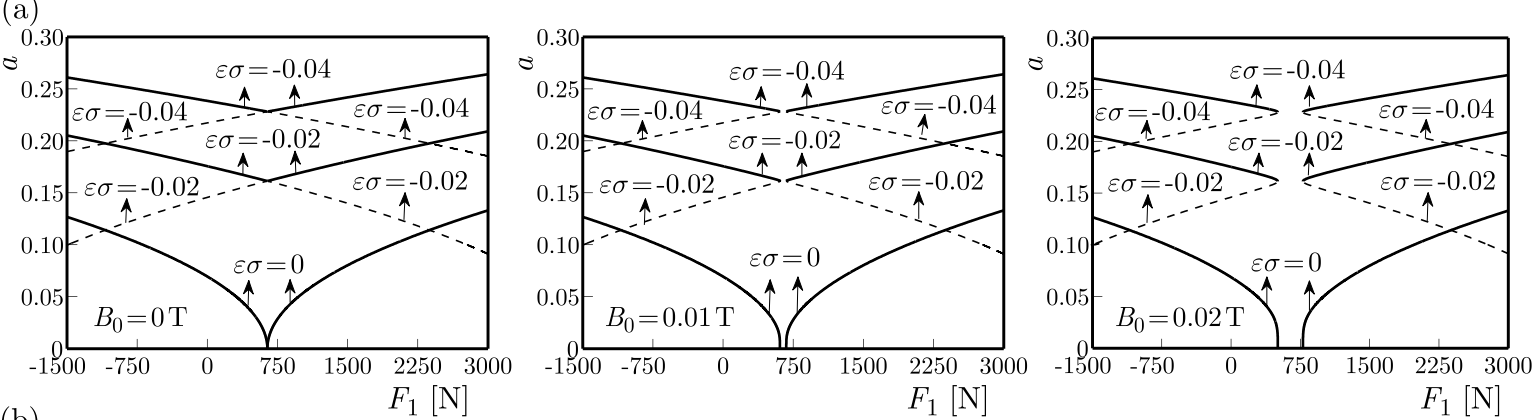

(b)
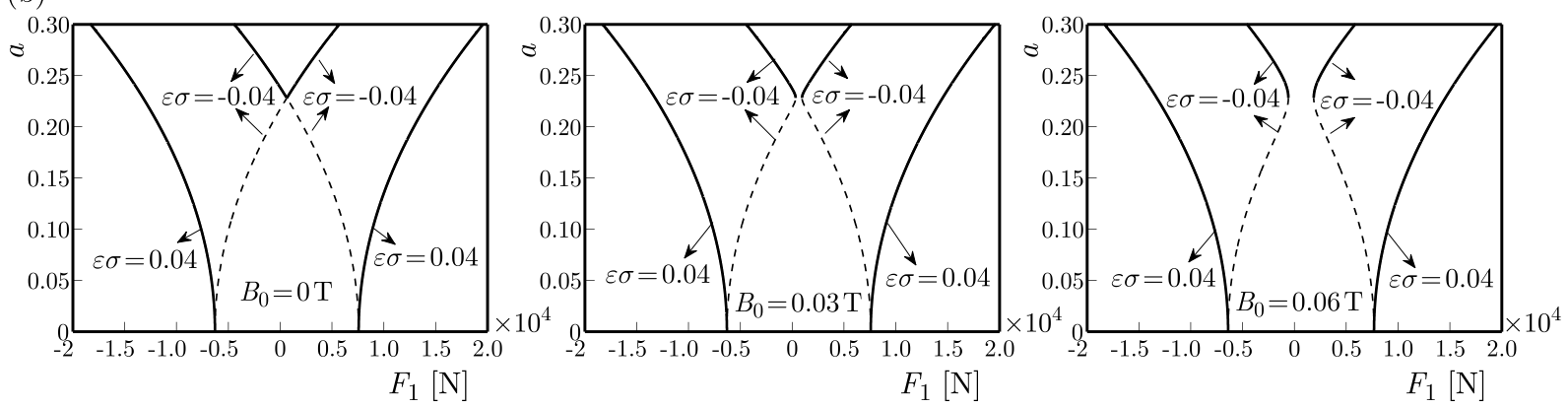

Fig. 5. The curve of the amplitude-parametric excitation, $F_{0}=30 \mathrm{kN}, J_{c}=0.02 \mathrm{~A} / \mathrm{mm}^{2}, P_{c}=0 \mathrm{~N} / \mathrm{m}$ :

(a) $C_{0}=60 \mathrm{~m} / \mathrm{s}, C_{1}=3 \mathrm{~m} / \mathrm{s}$ when $\varepsilon \sigma=0,-0.02$ and $-0.04 ;$ (b) $C_{0}=60 \mathrm{~m} / \mathrm{s}, C_{1}=3 \mathrm{~m} / \mathrm{s}$ when $\varepsilon \sigma=0.04$ and -0.04

decreases. In Figs. 7a, 7b and 7c, the curves become leveling off when the static load $Q$ increases to a certain value. The changes of the amplitude of the time-variant axial speed $C_{1}$ and the amplitude of the time-variant axial tension $F_{1}$ have a more significant effect on the vibration amplitude than that of the magnetic induction intensity $B_{0}$. 
(a)

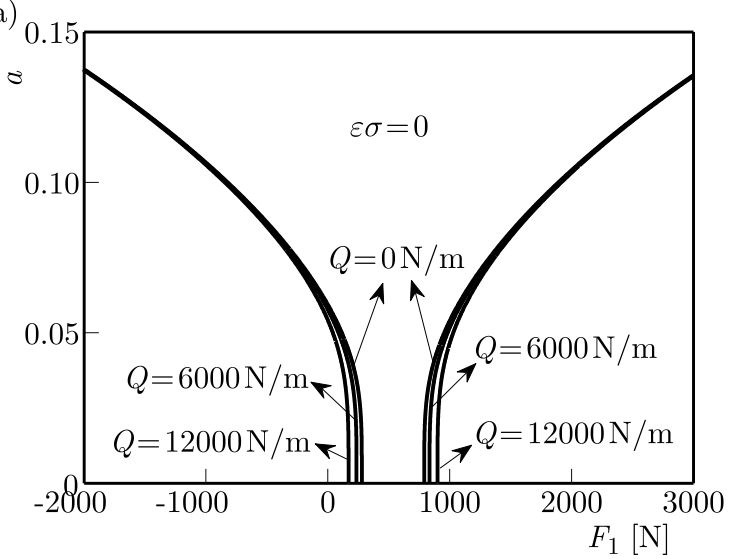

(b)

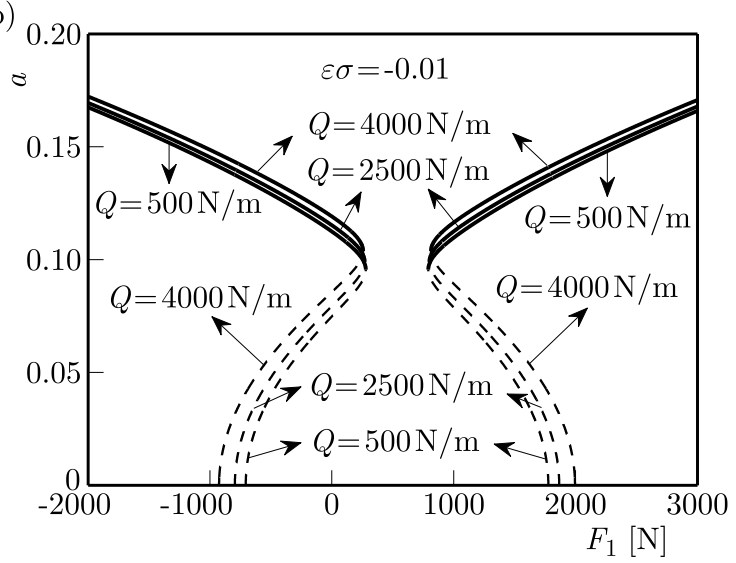

Fig. 6. The curve of amplitude-parametric excitation, $F_{0}=15 \mathrm{kN}, J_{c}=0.02 \mathrm{~A} / \mathrm{mm}^{2}$ :

(a) $C_{0}=50 \mathrm{~m} / \mathrm{s}, C_{1}=3 \mathrm{~m} / \mathrm{s}, B_{0}=0.03 \mathrm{~T}$; (b) $C_{0}=50 \mathrm{~m} / \mathrm{s}, C_{1}=3 \mathrm{~m} / \mathrm{s}, B_{0}=0.03 \mathrm{~T}$

(a)

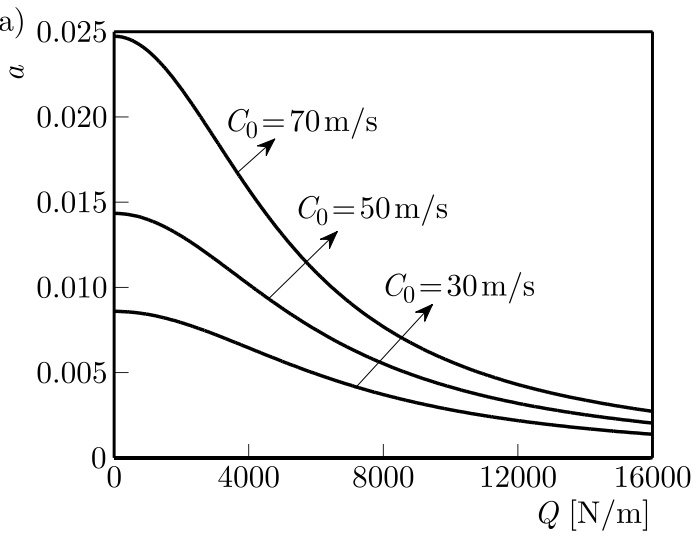

(c)

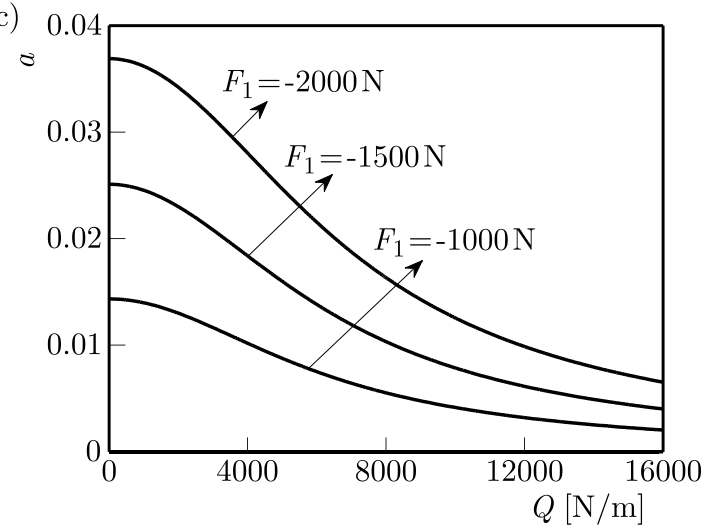

(b)

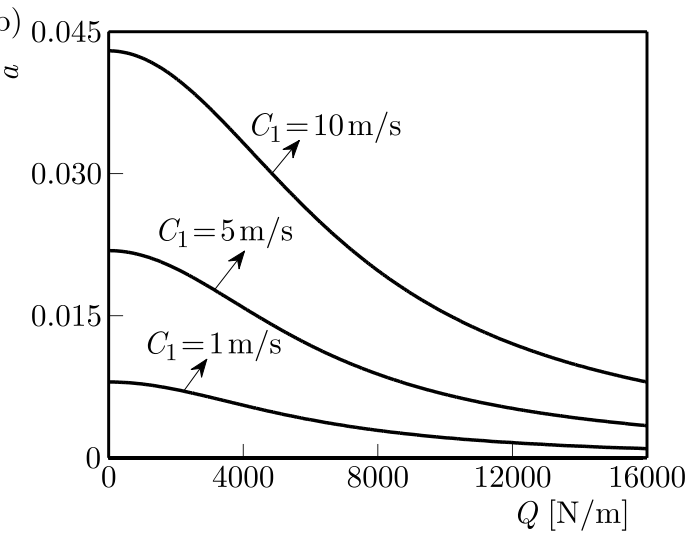

(d)

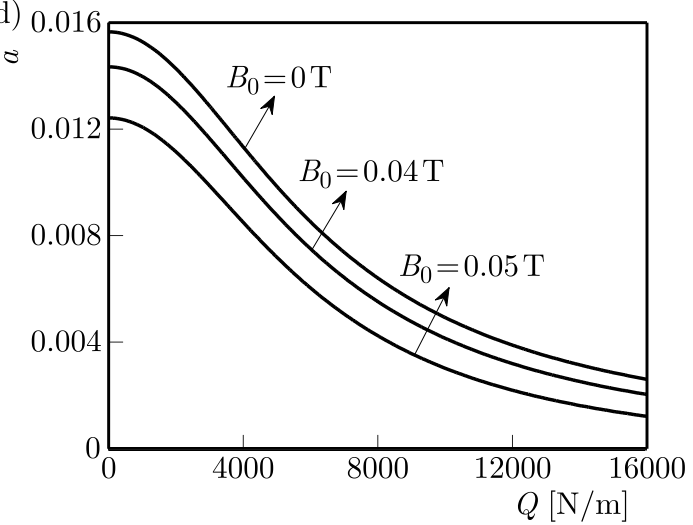

Fig. 7. The curve of amplitude - static load, $F_{0}=15 \mathrm{kN}, J_{c}=0.02 \mathrm{~A} / \mathrm{mm}^{2}, \varepsilon \sigma=0.01$ :

(a) $C_{1}=3 \mathrm{~m} / \mathrm{s}, F_{1}=-1000 \mathrm{~N}, B_{0}=0.04 \mathrm{~T}$; (b) $C_{0}=50 \mathrm{~m} / \mathrm{s}, F_{1}=-1000 \mathrm{~N}, B_{0}=0.04 \mathrm{~T}$;

(c) $C_{0}=50 \mathrm{~m} / \mathrm{s}, C_{1}=3 \mathrm{~m} / \mathrm{s}, B_{0}=0.04 \mathrm{~T}$; (d) $C_{0}=50 \mathrm{~m} / \mathrm{s}, C_{1}=3 \mathrm{~m} / \mathrm{s}, F_{1}=-1000 N$

\subsection{The bifurcation diagram of the amplitude}

In Fig. 8, the critical point of excitation is the intersection point of the trivial solution and resonance solutions (including both the stable and unstable solutions) of the system. As the figures show, in the upper region of each curve, the system has both a stable and an unstable solution, and in the lower part, the system has no solution. In the given range of the amplitude of the time-variant axial speed $C_{1}$, the time-variant axial tension $F_{1}$ of critical bifurcation increases with the growth of the magnetic induction intensity $B_{0}$. Given a certain range for $B_{0}$, the time-variant axial tension $F_{1}$ shows the same result as $C_{1}$ increases. 

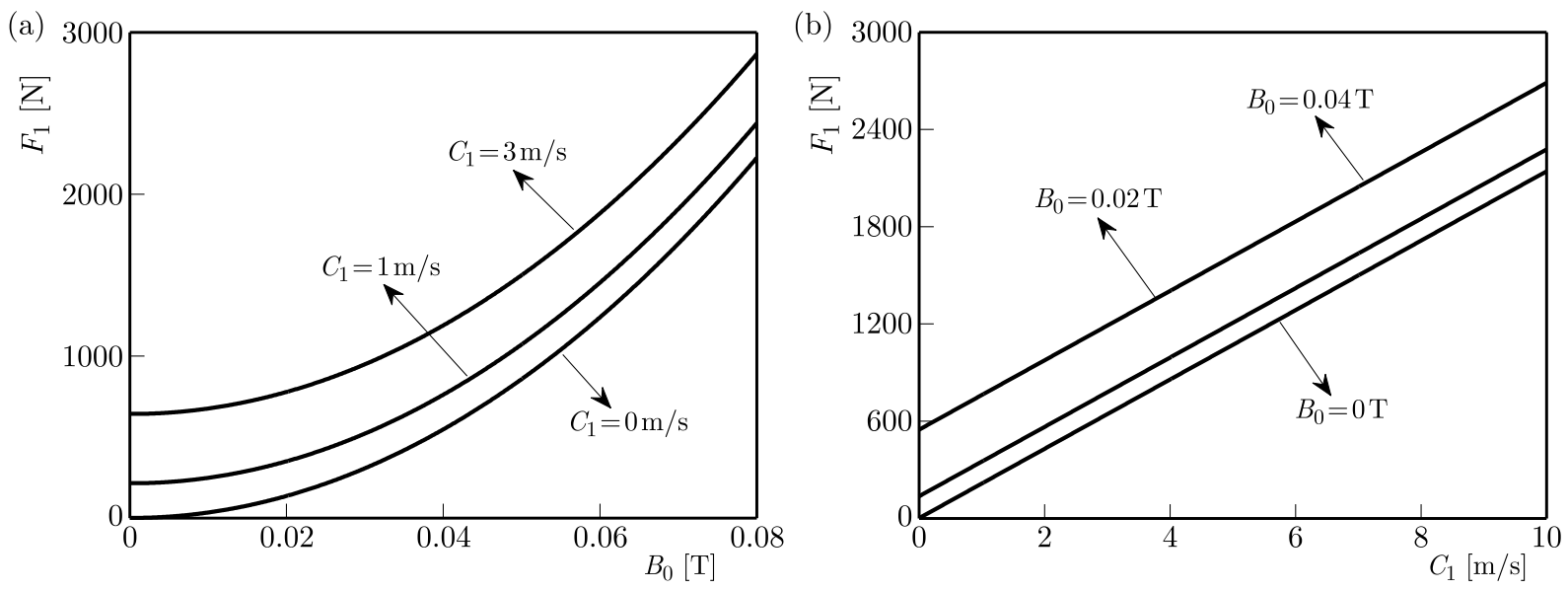

Fig. 8. The bifurcation diagrams of the amplitude, $C_{0}=60 \mathrm{~m} / \mathrm{s}, F_{0}=30 \mathrm{kN}, J_{c}=0.02 \mathrm{~A} / \mathrm{mm}^{2}$, $P_{c}=0 \mathrm{~N} / \mathrm{m}$ and $\varepsilon \sigma=-0.01$ : (a) $F_{1}-B_{0}$ bifurcation diagram of the amplitude; (b) $F_{1}-C_{1}$ bifurcation diagram of the amplitude

In Fig. 8a, the curves which correspond to Figs. 3c and 3d, show a rising trend with the increase of the amplitude of the time-variant axial speed $C_{1}$. The bifurcation diagrams of the time-variant axial tension $F_{1}$ versus the axial constant velocity $C_{0}$, which correspond to Figs. $4 \mathrm{~d}$, $4 \mathrm{e}$ and $4 \mathrm{f}$, are distributed in a parallel line pattern with a change of the magnetic induction intensity $B_{0}$ in Fig. 8b.

\section{Conclusions}

In this article, the primary parametric resonance and stability of an axially accelerating current-carrying beam under magnetic field are investigated. The effects of the detuning parameter, axial velocity, axial tension, magnetic induction intensity and static load on nonlinear characteristics of the system are discussed. The following conclusions can be drawn:

- The vibration amplitude varies with the physical parameters, and the system exhibits typical primary parametric resonance characteristics.

- The external magnetic field has a significant effect on the vibration of the system, and the vibration amplitude control can be achieved by controlling the value of magnetic induction intensity.

- When the axial time-variant speed is not zero, the symmetry axis of the resonant characteristic curve shifts towards right.

- The vibration amplitude under parametric resonance decreases with an increase of the static loads.

\section{Acknowledgment}

This work was supported by the Project of National Natural Science Foundation of China (No. 11472239), Hebei Provincial Natural Science Foundation of China (No. A2015203023) and the Key Project of Science and Technology Research of Higher Education of Hebei Province of China (No. ZD20131055).

\section{References}

1. Chakraborty G., Mallik, A.K., 1998, Parametrically excited non-linear traveling beams with and without external forcing, Nonlinear Dynamics, 17, 4, 301-324 
2. Chen L.Q., Tang Y.Q., Lim C.W., 2010, Dynamic stability in parametric resonance of axially accelerating viscoelastic Timoshenko beams, Journal of Sound and Vibration, 329, 5, 547-565

3. Chen L.Q., YAng X.D., 2005, Steady-state response of axially moving viscoelastic beams with pulsating speed: comparison of two nonlinear models, International Journal of Solids and Structures, 42, 1, 37-50

4. Ghayesh M.H., Balar S., 2008, Non-linear parametric vibration and stability of axially moving visco-elastic Rayleigh beams, International Journal of Solids and Structures, 45, 25-26, 6451-6467

5. Ghayesh M.H., Balar S., 2010, Non-linear parametric vibration and stability analysis for two dynamic models of axially moving Timoshenko beams, Applied Mathematical Modelling, 34, 10, 2850-2859

6. Hasanyan D.J., Khachaturyan G.M., Piliposyan G.T., 2001, Mathematical modeling and investigation of nonlinear vibration of perfectly conductive plates in an inclined magnetic field, Thin-Walled Structures, 39, 1, 111-123

7. Hasanyan D., Librescu L., Qin Z., Ambur D.R., 2005, Nonlinear vibration of finitely-electroconductive plate strips in an axial magnetic field, Computers and Structures, 83, 15, $1205-1216$

8. Hu Y.D., Hu P., Zhang J.Z., 2015, Strongly nonlinear subharmonic resonance and chaotic motion of axially moving thin plate in magnetic field, Journal of Computational and Nonlinear Dynamics, 10, 2, 1-12

9. Hu Y.D, Li J., 2009, The magneto-elastic subharmonic resonance of current-conducting thin plate in magnetic filed, Journal of Sound and Vibration, 319, 3, 1107-1120

10. Hu Y.D., WANG T., 2015, Nonlinear resonance of the rotating circular plate under static loads in magnetic field, Chinese Journal of Mechanical Engineering, 28, 6, 1277-1284

11. Hu Y.D., Zhang J.Z., 2013, Principal parametric resonance of axially accelerating rectangular thin plate in magnetic field, Applied Mathematics and Mechanics (English Edition), 34, 11, $1405-1420$

12. MAtsner V.I., 1978, Effect of initial deflections on the natural vibration frequencies of shells under axial compressive loads, Soviet Applied Mechanics, 14, 5, 528-531

13. NAYFeh A.H., Mook D.T., 1979, Nonlinear Oscillations, Wiley, New York

14. TAng Y.Q., Chen L.Q., YAng X.D., 2009, Parametric resonance of axially moving Timoshenko beams with time-dependent speed, Nonlinear Dynamics, 58, 4, 715-724

15. WANG J.Y., Chen K.J., 1993, Vibration problems of flexible circular plates with initial deflection, Applied Mathematics and Mechanics (English Edition), 14, 2, 177-184

16. WAng X.Z., Lee J.S., Zheng X.J., 2003, Magneto-thermo-elastic instability of ferromagnetic plates in thermal and magnetic fields, International Journal of Solids and Structures, 40, 22, $6125-6142$

17. Zheng X.J, Zhang J.P., Zhou Y.H., 2005, Dynamic stability of a cantilever conductive plate in transverse impulsive magnetic field, International Journal of Solids and Structures, 42, 8, 2417-2430 